\title{
An Efficient Integrated Routing Protocol for Interconnecting Mobile Ad Hoc Network and the Internet
}

\author{
Khaleel Ur Rahman Khan, Prof. A Venugopal Reddy, Rafi U Zaman
}

\begin{abstract}
There has been a massive growth in wireless communications in recent years. Many wireless communication techniques are commercially available like Wireless LAN, Bluetooth, and GSM to name a few. Access to the Internet via wireless communication devices has become an important issue due to the trend of an all-IP network in the 4G wireless networks. One such wireless technology gaining popularity is Wireless Mobile ad hoc Network, which is an infrastructure-less, autonomous, stand alone and self organizing network. It can be simply and flexibly deployed in almost any environment. However it has limited wireless coverage and limited connectivity. Mobile IP and IP micro-mobility protocols enable the mobile host to access Internet and change points of attachment without losing the connection. With the integration of MANET routing protocol and IP protocol, Internet connectivity to the MANET nodes can be achieved. Solutions to the integration problem, introduce an intermediate entity with hybrid mechanisms enabling it to connect to two different networks.

In this paper, an extended DSDV protocol, named as Efficient DSDV (Eff-DSDV) protocol has been used to provide bidirectional connectivity between ad hoc nodes and the hosts in the Infrastructure-based networks. The proposed framework uses one of the ad hoc hosts known as Mobile Internet Gateway (MIG) to act as a bridge between the two networks. The detailed performance comparison has been made between the proposed approach using Eff-DSDV routing protocol and then using the regular DSDV protocol. The simulation results not only show that the performance of the proposed strategy using Eff-DSDV is superior to using DSDV but also it is better than other integration strategies.
\end{abstract}

Index Terms- Eff-DSDV, Integration Strategy, Mobile ad hoc Network (MANET), Mobile Internet Gateway (MIG)

\section{INTRODUCTION}

Mobile ad hoc Network (MANET) is a wireless network which is created dynamically without the use of any existing network infrastructure or centralized administration [1]. The MANET nodes communication is limited to the Ad hoc Network only. Several applications may require a connection

Manuscript received on August 18, 2008.

Khaleel Ur Rahman Khan is with the CSE Department, Muffakham Jah College of Engg. And Tech., Hyderabad, INDIA (e-mail: khaleelrkhan@mjcollege.ac.in).

Prof. A. Venugopal Reddy is with the CSE Department, College of Engineering, Osmania University, Hyderabad, INDIA (e-mail: avgreddy@osmania.ac.in).

Rafi U Zaman is with the CSE Department, Muffakham Jah College of Engg. And Tech., Hyderabad, INDIA (e-mail: rafi.u.zaman@gmail.com). to an external network such as Internet or LAN to facilitate the users with the resources present in the external network. One such scenario could be that the members of a conference have formed an Ad hoc network to exchange data among themselves. But for another application, they need a connection to the Internet. For such a typical scenario, interconnecting the Ad hoc Network protocols and the protocols used in the infrastructure based network is needed. Other potential applications are remote surveillance or planetary exploration where a set of autonomous mobile robots collaboratively explore the surface of the distant planet, relaying telemetry information to one or more orbiting satellites part of inter-planetary Internet [2]. In infrastructure networks, a base station serves as a bridge for mobile hosts. Mobile users obtain their services under the coverage of a base station. The base station has both a wired and wireless interface and acts as a gateway between two types of networks [3]. On the contrary, Ad hoc nodes are mobile nodes connected without any such centralized infrastructure. Integrating $\mathrm{Ad}$ hoc networks with infrastructure networks can be beneficial for extending the coverage of wireless networks via multi hop communication of ad hoc hosts. It can also eliminate dead zones in wireless LAN [4]. Two important issues for providing the global Internet connectivity for Ad hoc networks are full duplex communication, reducing the Mobile IP [5] overhead, and the routing protocol overhead.

In this paper, the MANET and the infrastructure based network are integrated into a hybrid network. The main objective is to provide a full duplex connectivity between the Ad hoc hosts and the hosts on the infrastructure network, using the Efficient Destination Sequenced Distance Vector routing protocol (Eff-DSDV) [6]. One of the Ad hoc hosts is used as Mobile Internet Gateway (MIG) acting as a bridge between the MANET and the infrastructure network. The Full Duplex communication between MANET nodes and the Internet host is through this MIG. The MIG accesses the Switch in the wired network through an Access Point. The MIG is placed in close proximity to the Access Point. The MIG runs the Eff -DSDV protocol and takes care of the addressing mechanisms to ensure the transfer of packets between the hybrid networks. The addressing structure of the MANET nodes and the wired nodes may or may not be the same. MANET nodes may use flat addressing structure and the wired networks use hierarchical structure like IP addressing.

The rest of the paper is organized as follows. Section 2 
discusses the previous work done in this area. Section 3 given as overview of Mobile IP and DSDV [7]. Section 4 describes the basic model of the framework. Section 5 gives the design of the strategy by highlighting the Eff-DSDV protocol. Section 6 describes the experimental configuration and discusses the experimental results. Section 7 concludes the paper.

\section{PREVIOUS WORK}

The Integration strategies are generally classified as two-tier or three-tier architectures. The gateways can be fixed or mobile. The gateway discovery can be done in a proactive, reactive or hybrid approach. The gateway switching mechanisms can be different. Different MANET routing protocols are used. The detailed survey and issues can be found in [4].

- Lei and Perkins [8] proposal for integrating Ad hoc Networks and Internet, makes use of a Routing Information Protocol (RIP) within the ad hoc network.

- Broch and Johnson [9] proposed a solution to the integration of MANET with Mobile IP. It makes use of Border Gateway with two interfaces. One is configured with the normal IP, which is connected to the Internet. While the other connected to the MANET uses DSR protocol to route packets within MANET.

- In the proposal of Jönsson [10], called MIPMANET provides Internet access to the mobile nodes by making use of Mobile IP with Foreign Agent and reverse tunneling concept. MIPMANET makes use of AODV[11] protocol for routing of packets within the mobile nodes and the Foreign Agent. It makes use of MIPMANET Switching algorithm to decide whether a mobile node should change its Foreign Agent or not.

- Sun's proposal [3] makes use of AODV and Mobile IP to enable Internet connectivity to Mobile nodes. AODV is used for routing within the MANET, while Mobile IP is used for assigning care of address to the Mobile nodes. Hand off occurs only if a mobile node has not heard for more than one beacon interval.

- Ratanchandani and Kravets [12], has given a hybrid scheme to provide Internet connectivity to the MANET nodes, using Mobile IP. The scheme uses techniques such as TTL scoping of agent advertisements, eavesdropping and caching agent advertisements to combine the advantages of proactive and reactive approaches to providing connectivity.

- Tseng [13] proposal of the Integration and Implementation is based on IEEE 802.11b wireless LANs. Issues like overlapping of MANETs, dynamic adjustment of mobile agent's service coverage's, support of local broadcast and various communication scenarios are addressed.

- Habib Ammari [14], approach of integrating the MANET with Internet is based on the use of mobile Gateways. The mobile Gateways use Mobile IP when communicating with the Internet and DSDV when they interact with MANET nodes.

- Common Gateway Architecture [15], introduces a novel approach of having a single gateway through which the mobile nodes access the internet. The main feature of this scheme is that a single address space is used so that all the mobile nodes belonging to the MANET share the same address prefix.

- Denko [16] introduces a three-tier architecture using mobile gateways. An extended AODV protocol is used as the MANET routing protocol. Mobile nodes can maintain links to multiple mobile gateways using hybrid gateway discovery mechanisms.

- Rakesh Kumar [17], propose an efficient proactive gateway discovery algorithm that takes into account the size of the interface queue in addition to the traditional minimum hop metric to select an efficient gateway.

The Full Duplex Internet connectivity for mobile nodes is especially important in the integrated environment. The majority of the existing approaches has not clearly addressed the implementation of bi-directional connectivity. Moreover they have high packet latency, due to high route discovery latency. The integration of the mobile IP with proactive protocol facilitates for providing bi-directional connectivity. In our paper, an extended DSDV protocol named as Eff-DSDV has been used which improves the performance of the regular DSDV protocol [6]. In our strategy one of the Ad hoc hosts using Eff-DSDV acts as Foreign Agent apart from serving as mobile IP proxy for the Ad hoc nodes.

\section{PRELIMINARIES}

\section{A. Mobile IP}

The Mobile IP is defined by IETF to support mobility [5]. It is transparent to TCP and UDP. There is no need to change the IP addresses when the mobile host moves from one subnet to the other. Mobile IP defines four important Terms: Mobile Host ( $\mathrm{MH})$, Correspondent Host $(\mathrm{CH})$ Home Agent (HA) and Foreign Agent (FA. A MH is a host or a router that may change its point of attachment from subnet to subnet. When a $\mathrm{CH}$ sends an IP datagram to a $\mathrm{MH}$, it will be delivered to the MH's home network. When the $\mathrm{MH}$ is away from home, the datagram will be tunneled to the foreign network. The HA will encapsulate the datagram with an IP header carrying the FA's IP address or the MH's co-located care of address. In case of FA's address, the FA must de-capsulate the datagram and forward it to the MH. CoA may be dynamically obtained through DHCP protocol. In case of $\mathrm{CoA}$, the $\mathrm{MH}$ itself acts as the endpoint of the tunnel whereupon it performs the de-capsulation process. Generally the former approach is used. HA and FA need to advertise their services by periodically sending AGENT-ADVERTISEMENT. A MH unaware of any local mobile agent may enquire by sending AGENT_SOLICITATION. From time to time, MH needs to register with its HA its current CoA. HA maintains the list of all registered MH's (its permanent address and CoA) in a location dictionary.

\section{B. Destination Sequenced Distance-Vector (DSDV) Protocol}

The DSDV [7] is one of the first protocols proposed for wireless Ad hoc Networks. It is an enhanced version of the distributed Bellman-Ford Algorithm, wherein each node maintains a table that contains the minimum distance and the 
first node on the shortest path to every destination node in the network. The Table updates are done with increasing sequence number tags in order to prevent loops, to deal with count-to-infinity problem, and for faster convergence. Routes to all destinations are available at every node at all times. The tables are exchanged at regular intervals of time so that an up-to-date view of the network topology is maintained. The Tables are also forwarded if a node observes a significant change in local topology. The table updates can either be incremental or full dumps. Incremental updates taking a single NPDU, is done when the node does not observe significant changes in the local topology. A full dump taking multiple NPDUs is done when there is a significant change in the topology. The table updates are initiated by the destinations with a new sequence number which is always greater than the previous one. Upon receiving the table a node may either update its table or may wait for another table from its next neighbor. Based on the sequence number of the table update, it may forward or reject the table.

The reconfiguration of a path used by an on-going data transfer session is handled as follows: The end node of the broken link initiates a table update message with the broken link weight assigned to infinity and with a sequence number greater than the stored sequence number for that destination. Each node, upon receiving an update with weight infinity, disseminates it to its neighbours in order to propagate link information to the whole network. Thus a single link break leads to the propagation of table update information to the whole network. A node always assign an odd sequence number to the link break update, to differentiate it from the even sequence number generated by the destination.

One of the drawbacks of this protocol is, the updates due to broken links lead to a heavy control overhead during high mobility. Even a small network with high mobility or a large network with low mobility can choke the bandwidth. Another drawback is the stale routes: In order to obtain information about a particular destination node, a node has to wait for a table update message initiated by the same destination node.

\section{MODEL OF THE CONNECTIVITY FRAMEWORK}

The objective of the framework is to integrate the Ad hoc Network and the infrastructure (wired) network by means of the mobile hosts, which are located under the radio range of Mobile Internet Gateway (MIG), which acts as the bridge between the hybrid networks. The Ad hoc routing protocol Eff-DSDV and Mobile IP coordinate with each other to provide the connectivity across the hybrid network as shown in the Figure 1. The Figure 2 shows the protocol architecture used in the framework. It shows the layers of protocols used in the MIG, HA, CN, Ad hoc node.

There are two ways for the mobile host to obtain the global connectivity. In the first scenario, the mobile host may be directly under the coverage of the MIG i.e. one hop away from MIG, be able to send its data directly to the MIG. In the second case, the ad hoc hosts which are outside the range of the MIG communicate with the MIG using multi-hop communication links. One of the important objectives of the framework is to minimize the overhead of MIP and Eff-DSDV. Figure 1 shows the communication scenario

between the two networks. The left side of the figure shows the infrastructure network having the Home Agent, Correspondent node, MIG. The right side has the ad hoc hosts using the routing protocol. Every ad hoc host registers with the HA and creates a binding at HA by mapping its home address to the care of address (COA) provided by the MIG. It is once again reminded that the MIG acts as the FA for all the ad hoc hosts. After receiving the data packets sent by the $\mathrm{CN}$, the router tunnels them to the MIG/FA. Then the MIG delivers the packets to the ad hoc host using the Eff-DSDV protocol. On the other hand, after receiving the data packets sent by the ad hoc host, the MIG delivers them to the $\mathrm{CN}$ thru the router using the IP routing in the infrastructure network.

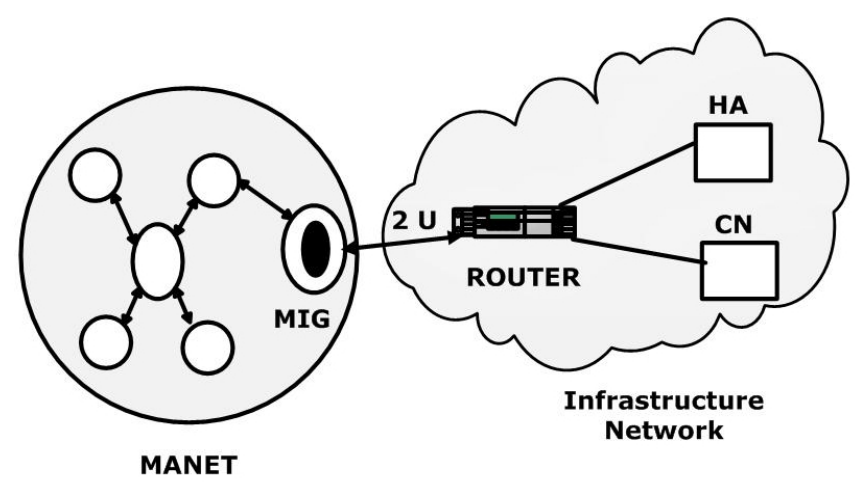

Fig. 1 Basic Model of the Connectivity Framework

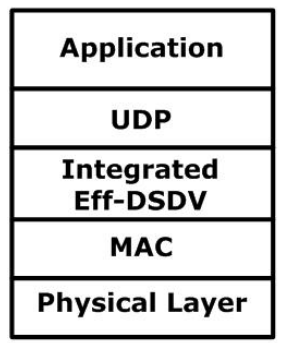

AdHoc Node

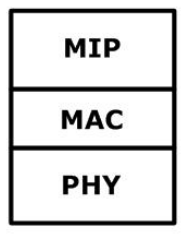

Home Agent (HA)

\begin{tabular}{|c|c|}
\hline Application & Application \\
\hline UDP & UDP \\
\hline $\begin{array}{c}\text { Integrated } \\
\text { Eff-DSDV }\end{array}$ & MIP \\
\hline MAC & MAC \\
\hline PHY & PHY \\
\hline
\end{tabular}

Mobile Internet Gateway (MIG)

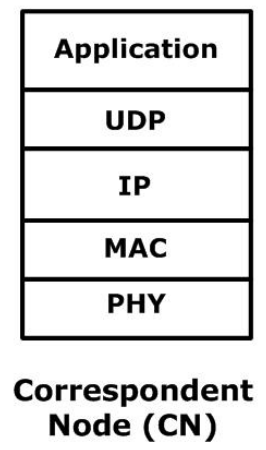

Fig. 2 Protocol Architecture of the Framework

\section{DESIGN OF THE CONNECTIVITY FRAMEWORK}

\section{A. Efficient DSDV Protocol (Eff-DSDV)}

In DSDV protocol, each mobile host maintains a routing table that stores the number of hops, and the sequence number for all the destinations. The routing table updates may be time-driven or event-driven. The interval between the two updates is known as the periodic route update interval. In 
DSDV the low packet delivery is due to the fact that, it uses stale routes in case of broken links. In DSDV the existence of stale route does not imply that there is no valid route to the destination. The packets can be forwarded thru other neighbors who may have routes to the destination. When an immediate link from the host say ' $S$ ' to the destination say ' $\mathrm{T}$ ' breaks, the proposed protocol creates a temporary link thru a neighbor which has a valid route to the desired destination. The temporary link is created by sending one-hop ROUTE-REQUEST and ROUTE-ACK messages. The host say ' $S$ ' upon finding the next hop broken link broadcasts a one-hop ROUTE-REQUEST packet to all its neighbors. In turn, the neighbors returns the ROUTE-ACK if it has a valid route to the destination and the host ' $S$ ' is not the next hop on the route from the neighbor to the destination. Each entry in the routing table has an additional entry for route update time. This update time is embedded in the ROUTE-ACK packet and is used in selecting a temporary route. In case of receiving multiple ROUTE-ACK with the same number of minimum hops, ad hoc host ' $S$ ' chooses that route which has the latest update time. Therefore, it may be surmised that the proposed Eff-DSDV follows the conventional DSDV but reduces the packet loss due to broken links. As shown in our paper [6], the performance of Eff-DSDV is superior to DSDV in terms of packet delivery ratio, end-end delay and the number of dropped packets. The mechanism of Eff-DSDV is explained by the following algorithm.

Algorithm Eff-DSDV (Host A, Destination D, MAXBufferSize N, Packet X)

1. if (A. NextHopLink ()$==$ ACTIVE) then Use Standard DSDV; else if (A. BufferLength ()$==N$ ) then Discard X; else

place $\mathrm{X}$ in A.Buffer;

2. A. Broadcasts (ROUTE-REQUEST, 1, D, A);

3. if (A. NEXT_NEIGHBOR has route to 'D') then

\section{A. RECIEVE (ROUTE_ACK)}

4. Min_Hops $=\infty$; Next_Hop $=0$; Updated_Time $=0$;

5. While (Host A has ROUTE_ACK Packets) \{

if ( ROUTE_ACK.HOP_COUNT< $<$ =Min_Hops )

\{

if $($ ROUTE_ACK.HOP_COUNT =Min_Hops $)$

\{

if ( ROUTE_ACK.UPDTD_TIME >

Updated_Time )

\{

Host_ID = ROUTE_ACK.HOST_ID;

Updated_Time $=$ ROUTE_ACK.UPDTTD_TIME;

\}

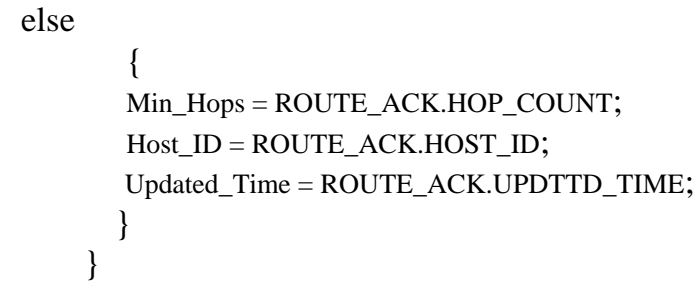

else

\{

Min_Hops = ROUTE_ACK.HOP_COUNT;

Host_ID = ROUTE_ACK.HOST_ID;

Updated_Time $=$ ROUTE_ACK.UPDTTD_TIME;$$
\}
$$$$
\text { \} }
$$

6. Forward the incoming Packet ' $\mathrm{X}$ ', via Host_ID;

If the next hop link of Host A for the required destination is active, then it uses the conventional DSDV Protocol.

In case if any outgoing link breaks, and if there is no capacity in the buffer, then the incoming packet is discarded. Otherwise the packet is buffered for later transmission. The alternate route discovery process starts.

The Host A broadcast a one-hop Route Request packet to its neighbors. If the next hop neighbor has a valid route to the destination in its routing table and if A is not the Next-Hop, then it sends Route Acknowledgement message enlisting its Host ID, the destination, the hop count metric for the destination and the last updated time.

The Host A (in the while loop) chooses the best neighbor, based on the least number of hops to the destination. If there is more than one node having the same number of hops, then it selects the host with the latest routing update time. The packets (buffered) are then forwarded using the latest found route till, the routing table of host $\mathrm{A}$ is updated by the conventional DSDV routing protocol.

\section{B. Full Duplex Connectivity between MANET and Wired Network}

In the proposed framework, MIG plays the role of FA. The MIG takes part in routing just as other Ad Host do. It also acts as Mobile IP Proxy for them. The MIG doesn't broadcast the Agent advertisements for the purpose of registration of the hosts. This broadcast is the source of the overhead in the other strategies namely $[3,9,12]$. In the proposed protocol, ad hoc hosts and the MIG know each others presence via routing update of the Eff-DSDV protocol. Whenever the Ad hoc host say "A" joins the ad hoc network, the host A broadcasts DSDV advertisements to its neighbors with sequence number of ' 0 '. Each host takes a note of it and makes an entry about host " $A$ " in their routing tables. Later they broadcast with increased sequence number to their neighbors. This broadcasting process continues until the advertisements reach all the destinations i.e the diameter of the network. The MIG also comes to know about the host "A" and makes an entry in its routing table. The host " $\mathrm{A}$ " also gets routing updates from its neighbors and thereby creates its routing table, including the route to MIG. Thus all the nodes know routes to every other node in the network including the MIG. After this, the host "A" sends its registration information to the MIG like its Home Address. Based on this information MIG acts as a mobile IP proxy for "A". The MIG sends the registration request to the host's Home Agent. After successful registration, a registration reply is returned to the MIG from the HA. The MIG then informs the host " $\mathrm{A}$ " about 
the registration status. The MIG keeps the registration information of all the ad hoc hosts and uses it during re-registration. The MIG uses its address as the COA to register with the HA. The mobile IP registration lifetime is taken as 3 times that of DSDV periodic route update interval.

\section{- MIG Registration Maintenance}

The advantage of the proposed strategy is that the ad hoc hosts doesn't have to participate in the mobile IP registration, because MIG keeps all the registration information for each node. The MIG updates the registration for each ad hoc host before its expiration only if the MIG has a valid route to the ad hoc host. Therefore the MIG has the complete and consistent view of the entire network. It knows whether or not an ad hoc host is part of the network or not. In case if any ad hoc node roams away, then the hop count metric for the ad hoc host becomes infinite in the routing table of MIG. If it loses the route to an ad hoc host for 3 times the period of the EDSDV update interval, then the MIG sends De-registration message to the HA of the roamed away host. If the host joins the network again, then the MIG initiates a fresh registration process.

In order to be able to communicate with both network types, the MIG uses the protocols of the wired network and the wireless ad hoc Network. The ad hoc node uses the protocol stack that is similar to the wired nodes except that it uses Eff-DSDV protocol to route packets. To commission two protocol stacks, MIG uses two wireless interfaces. The first one is configured for the infrastructure mode and has the protocol stack of normal wired nodes. With this interface the MIG is able to connect to the Access Point. This Access Point is the connection between wireless and wired communication. It converts from wired to wireless and the other way round. The other interface of the MIG is configured for the ad hoc mode and has the Eff-DSDV ad hoc routing protocol

\section{Communication Scenarios in the proposed Framework}

The proposed framework provides the following three modes of communication in order to provide full duplex communication:

B Communication within the MANET (Intra-MANET routing): This form of communication is the conventional communication within the ad hoc network by means of Eff-DSDV routing protocol. To send data to another host, the ad hoc host checks its routing table. If the destination is within the ad hoc network, then there will be an entry in the routing table leading to the destination. Accordingly the packets will be routed to the next-hop and continues till it reaches the destination.

B Communication from ad hoc Host to Correspondent Host: This type of communication happens between an ad hoc host and a host in the infrastructure network known as CN. The ad hoc host checks its routing table for the $\mathrm{CN}$ address. If no entry for such host is found, then it searches for the route to the MIG. If the route is found to MIG then the packets are forwarded to the MIG thru intermediate ad hoc nodes (if required) using Eff-DSDV routing protocol. Then the MIG noticing that, this packet's destination lies outside the network, processes the packet and then forwards it to the router thru the access point. The packet now will bear the destination IP address and sent to the destination as if it were attached to the normal IP subnet instead of DSDV ad hoc network. If the routing entry to the MIG is not found, then the packet is discarded.

ß Communication between Correspondent Node and an ad hoc Host: This type of communication is initiated by the $\mathrm{CN}$. The $\mathrm{CN}$, when wishing to send packets to the ad hoc host, delivers the packet to the HA. If the ad hoc host had registered with the HA, then it will forward the packet to the ad hoc host in consultation with its routing table. If the ad hoc host has roamed away from it's HA domain, then the HA will have the COA of MIG if it has registered. Using the CAO the packet will be tunneled to the MIG by the HA. The MIG checks its routing table, and delivers the packets to the requested destination ad hoc host using the Eff-DSDV routing protocol.

\section{Simulation Results AND ANALYSis}

\section{A. Simulation Setup}

The effectiveness of the proposed framework is evaluated by carrying out the simulation experiments, in which the ad hoc hosts and the MIG runs the Eff-DSDV routing protocol. The simulation experiments were conducted using NCTUns, network simulator [18]. It is one of the modern versatile simulators supporting the simulation of the most of the wired and wireless technologies including WiMAX, Vehicular communications. A snapshot of our simulation experiment is shown in Figure 3. The snapshot shows the ad hoc hosts, MIG, router, access points, $\mathrm{HA}$ and $\mathrm{CN}$. The parameters that are common to all the simulations are shown in Table 1.

TABLE I: SiMULATION PARAMETERS

\begin{tabular}{|l|l|}
\hline Parameter & Value \\
\hline Number of ad hoc Hosts & 20 \\
\hline Number of Packet(Traffic) Sources & 3 \\
\hline Number of MIG & 2 \\
\hline Number of CN & 1 \\
\hline Topology Size & $800 \times 800 \mathrm{~m}$ \\
\hline Transmission(radio) range & $250 \mathrm{~m}$ \\
\hline Traffic Type & $\begin{array}{l}\text { Constant Bit Rate } \\
\text { (CBR) }\end{array}$ \\
\hline Packet size & 512 Bytes \\
\hline Standard packet sending rate & $5 \mathrm{packets} / \mathrm{sec}$ \\
\hline Standard ad hoc host speed & $20 \mathrm{Kbps}]$ \\
\hline MIG speed & $20 \mathrm{~m} / \mathrm{s}$ (Max) \\
\hline Mobility Model & $4 \mathrm{~m} / \mathrm{s}$ \\
\hline Pause Time & Random waypoint \\
\hline Simulation Time & $5 \mathrm{sec}$ \\
\hline Wireless channel bandwidth & $400 \mathrm{sec}$ \\
\hline $\begin{array}{l}\text { Interface Queue Length(wireless \& wired } \\
\text { nodes) }\end{array}$ & $2 \mathrm{Mbps}$ \\
\hline Wired link bandwidth & $50 \mathrm{packets}$ \\
\hline Eff-DSDV periodic route update interval & $10 \mathrm{Mbps}$ \\
\hline $\begin{array}{l}\text { No. of time the Eff-DSDV ROUTE } \\
\text { REQUEST may be resent }\end{array}$ & $2 \mathrm{sec}$ \\
\hline $\begin{array}{l}\text { Time duration before sending a new } \\
\text { ROUTE REQUEST }\end{array}$ & $1 \mathrm{sec}$ \\
\hline
\end{tabular}




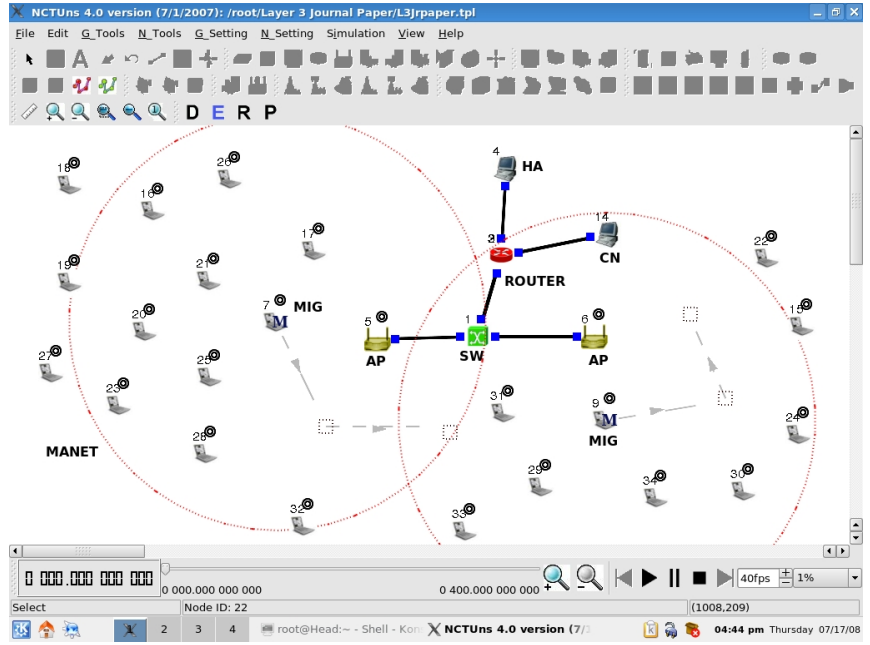

Fig. 3 Simulator snapshot

\section{B. Simulation Results Discussion}

The performance of the proposed framework is analyzed with respect o the following metrics.

- Packet delivery ratio: defined as the number of received data packets over the number of generated data packets.

- Eff-DSDV and Mobile IP overhead: defined as the number of the DSDV and Mobile IP messages.

- End- End delay: defined as the difference of the time a packet is received at the destination and time it is generated at the source.

The metrics have been obtained as the function of ad hoc node speed and as a function of the traffic load. For each sampled data point, NCTUns was executed three times under different random mobility scenarios and each data point shown in the graphs is an average of three results. The performance of the proposed approach has been compared by running the Eff-DSDV routing protocol and later the regular DSDV routing protocol, on the ad hoc hosts and the MIG.

Figure 4 to 7 shows the impact of the ad hoc host speed on the packet delivery ratio, routing protocol overhead, end-end delay and MIP overhead. As is seen in Figure 4, the packet delivery ratio goes down as the node speed increases from 5 to $25 \mathrm{~m} / \mathrm{s}$. The throughput is better than other integration approaches like [12] and the packet delivery ratio is higher in the case of Eff-DSDV than the regular DSDV protocol.

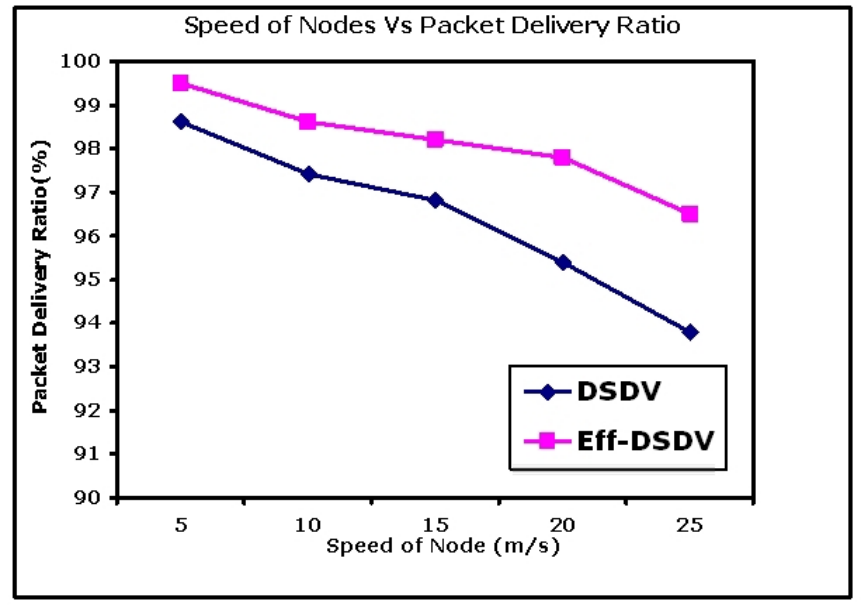

Fig. 4 Packet delivery ratio as a function of ad hoc host speed

Figure 5 and 6 indicates the routing protocol and MIP overhead as a function of the node speed. It is evident from Figure 6 that the MIP overhead in the proposed approach is very low. This is due to the fact that ad hoc hosts send their registration information to the MIG only at the time of joining the ad hoc network. There is no broadcast of the Agent advertisements. The routing protocol overhead is as well in control. It may be noted from Figure 6 that the routing protocol overhead of Eff-DSDV is slightly higher than the DSDV approach due to the broadcast of Route Request packet whenever the link breaks. These Route Request broadcasts are not present in the DSDV protocol.

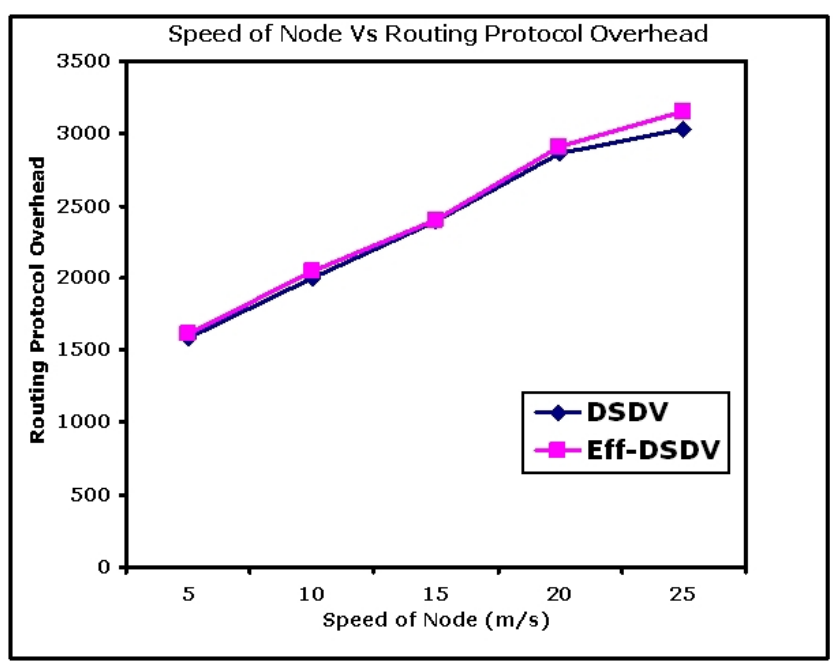

Fig. 5 Routing Protocol Overhead as a function of ad hoc host speed 


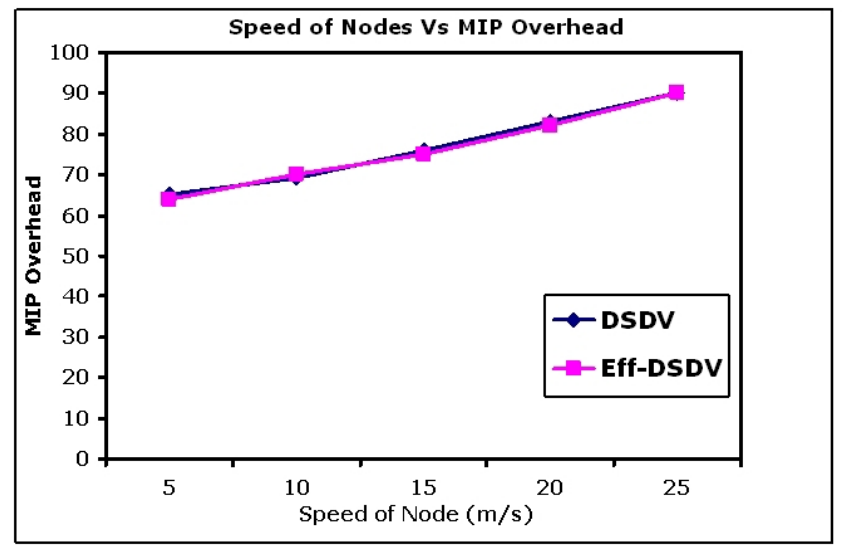

Fig. 6 Mobile IP Overhead as a function of ad hoc host speed

Figure 7 shows the impact of the node speed on the packet delay. It can be very clearly seen that the packet delivery latency is remarkably less using Eff-DSDV in comparison with regular DSDV routing protocol. The reason is that the Eff-DSDV uses temporary routes in the case of link failures and hence delivers the data faster than the DSDV which has to wait till all the routing table entries are stabilized.

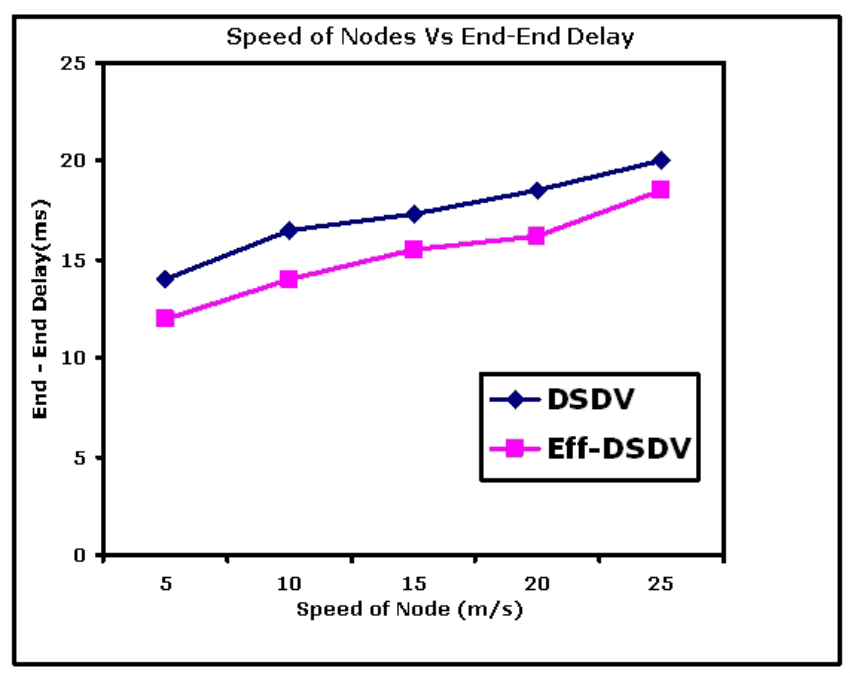

Fig. 7 End-End packet delay as a function of ad hoc host speed

The next set of graphs in Figures 8 to 11 shows the relationship of the packet delivery ratio, routing protocol overhead, MIP overhead and end-end delay with respect to packet load. The packet load is the number of packets transmitted per second per source. As shown in Figure 8, the packet delivery ratio is slightly higher for low packet load but it decreases with increase in the traffic load. Figure 9 and 10 shows routing protocol and MIP overhead respectively. It may be noted that there is no much difference between the Eff-DSDV and the DSDV protocol. Figure 11 shows, that the End-End delay increases as expected when the load increases This is due to more collisions, retransmissions and routing dumps.

It may be observed from the above graphs that, the proposed strategy using Eff-DSDV performs better than using DSDV protocol. It is also observed that the proposed approach even performs better than the approaches of [10, 12].This is due to the fact that, the total overhead for maintaining full connectivity for ad hoc hosts is higher in [10, 12] due to extra AODV messages which is not required in the proposed approach due to the EFF-DSDV protocol. The Eff-DSDV protocol uses better routes than AODV so that the packets can reach the destination earlier. Moreover, EFF-DSDV doesn't require time for route discovery since the routes already exists between the source and the destination. Whereas the route is created on demand basis in the case of using AODV protocol as in [10, 12].

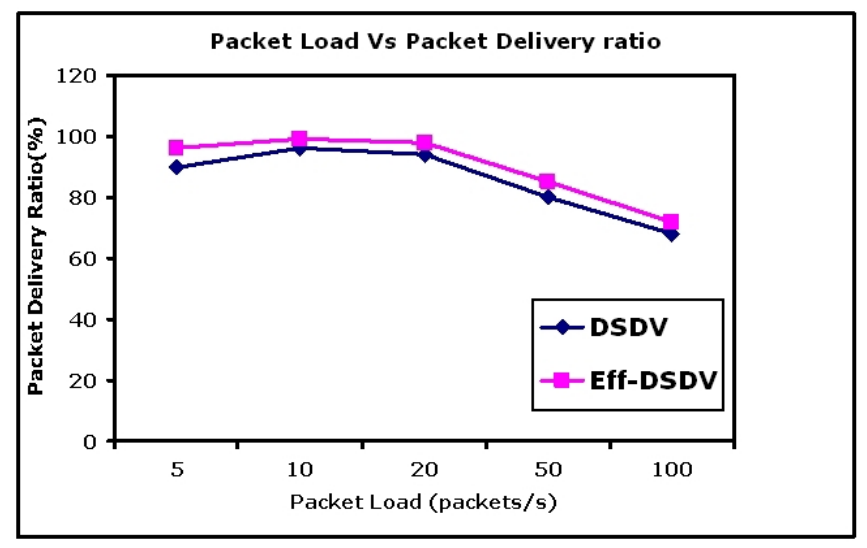

Fig. 8 Packet delivery ratio as a function of Packet load

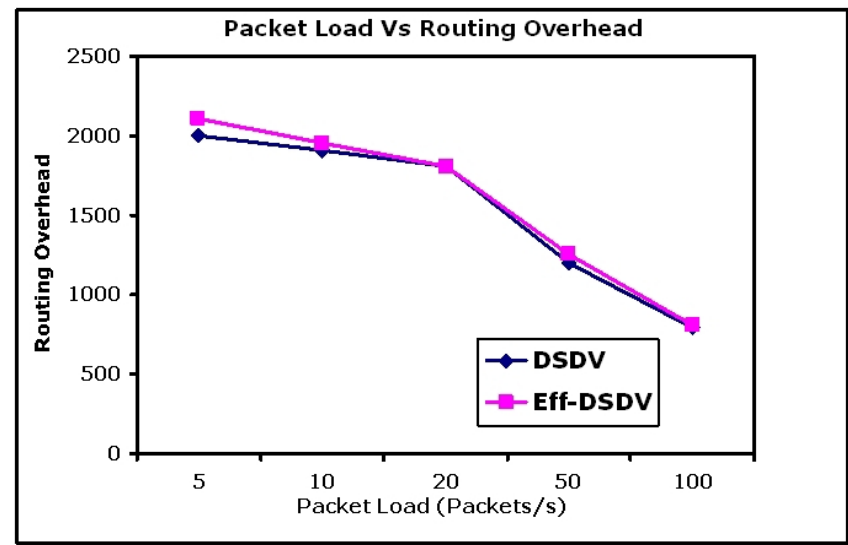

Fig. 9 Routing Protocol Overhead as a function of Packet load

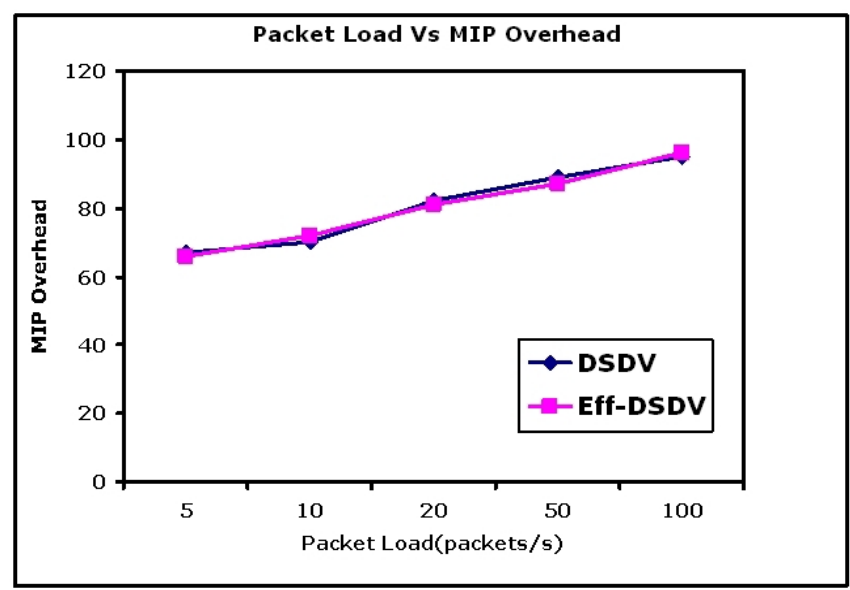

Fig. 10 Mobile IP Overhead as a function of Packet load 


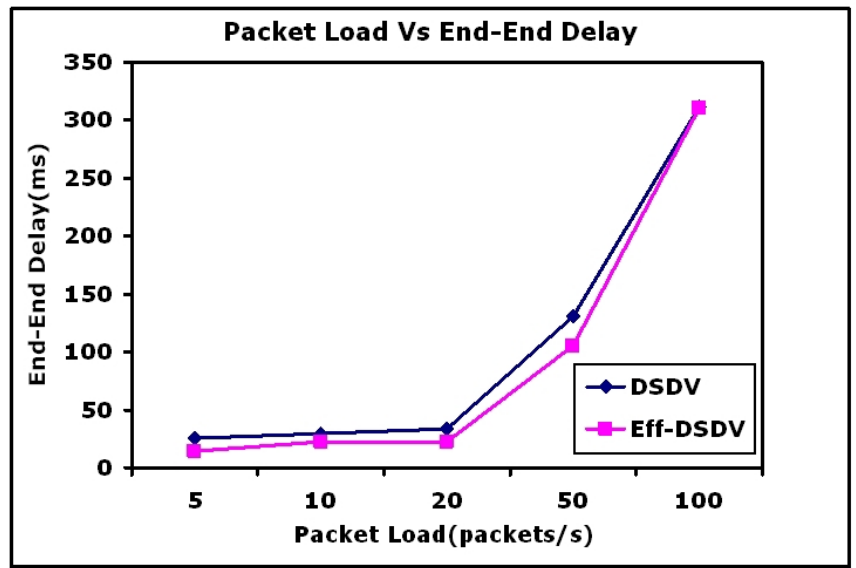

Fig. 11 End-End delay as a function of Packet load

\section{CONCLUSION}

We have presented a solution for integrating mobile ad hoc network running an Eff-DSDV routing protocol with the wired network. One of the distinguishing characteristics of this framework is that it uses one of the ad hoc hosts as the Mobile Internet Gateway (MIG) to interconnect the two networks. The framework provides full duplex connectivity for ad hoc Network nodes. One more advantage of this strategy is that it does not require the flooding of the Gateway advertisements for registration of ad hoc hosts with the MIG. The MIG and the ad hoc hosts learn about their presence due to the routing table exchanges. The simulation results indicate that the proposed strategy gives desirable results with respect to metrics packet delivery ratio, end-end delay, MIP and routing protocol overhead. The simulations results of the proposed approach using Eff-DSDV routing protocol have been compared with using DSDV protocol. The results clearly indicate the superior performance with Eff-DSDV over DSDV protocol. More Simulation experiments can be done evaluating the performance with respect to other metrics like Traffic load, Beacon intervals.

\section{REFERENCES}

[11] E.M. Royer, C-K. Toh, "A Review of Current Routing Protocols for ad hoc Mobile Wireless Networks," IEEE Personal Communications Magazine, April 1999, pp 46-55.

[12] Erik Nordstrom, P Gunningberg, C Tschudin, "Design of Internet Connectivity for Mobile ad hoc Networks, "Uppsala University.

[13] Y. Sun, E.M. Belding-Royer, C.E. Perkins, "Internet Connectivity for ad hoc Mobile Networks, "International Journal of Wireless Information Networks, Special Issue on Mobile ad hoc Networks(MANETs): Standards, Research, Applications, April 2002, pp 75-88.

[14] Khaleel Ur Rahman Khan, Rafi U Zaman, A.Venugopal Reddy, "Integrating Mobile ad hoc Networks and the Internet: challenges and a review of strategies, " Proceedings of IEEE/CREATE-NET/ICST COMSWARE 2008, January 2008.

[15] C.E. Perkins, “Mobile Networking Through Mobile IP, ” IEEE Internet Computing, Vol. 2, Issue 1, January 1998.

[16] Khaleel Ur Rahman Khan, Rafi U Zaman, A.Venugopal Reddy et. al "An Efficient Destination Sequenced Distance Vector Routing Protocol for Mobile ad hoc Networks, " Proceedings in IEEE Comp.Soc., of ICCSIT Singapore, September 2008, (Paper accepted).

[17] C.E. Perkins \& P. Bhagwat, "Highly Dynamic Destination Sequence-Vector Routing (DSDV) for Mobile Computers", Computer Communication Review, 24(4), 1994, 234-244.

[18] H. Lei and C.E. Perkins, ad hoc Networking with Mobile IP, Proceedings of the 2nd European Personal Mobile Communications Conference, Bonn, Germany, 1997, 197-202.
[19] J. Broch, D.A. Maltz, D.B. Johnson, "Supporting Hierarchy and Heterogeneous Interfaces in Multi-Hop Wireless ad hoc Networks, ” Proceedings of the Workshop on Mobile Computing, Perth, Australia, June 1999.

[20] U. Jonsson, F. Alriksson, T. Larsson, P. Johansson, G.Q. Maguire Jr., "MIPMANET - Mobile IP for Mobile ad hoc Networks," The First IEEE/ACM Annual Workshop on Mobile ad hoc Networking and Computing(MobiHOC), Boston, Massachusetts, USA, August 11, 2000, pp 75-85.

[21] C.E. Perkins and E.M. Royer, "Ad-Hoc on-Demand Distance Vector routing, " Proc. Workshop Mobile Computing Systems and Applications (WMCSA '99), Feb. 1999 pages 90-100.

[22] P. Ratanchandani, R. Kravets, "A Hybrid approach to Internet connectivity for Mobile ad hoc Networks, " Proceedings of IEEE Wireless Communications and Networking Conference (WCNC), New Orleans, Louisiana, USA, 16-20 March 2003, pp 1522-1527.

[23] Y.Tseng, C.Shen, W.Chen, "Mobile IP and ad hoc Networks: An Integration and Implementation Experience, ” IEEE Computer Vol. 36, No. 5, May 2003, pp 48-55.

[24] H. Ammari, H. El-Rewini, "Integration of Mobile ad hoc Networks and the Internet Using Mobile Gateways, " Proceedings of the 18th International Parallel and Distributed Processing Symposium (IPDPS '04) Workshop 12, 2004, pp 218b.

[25] M.Michalak, T. Braun, "Common Gateway Architecture for Mobile Ad-Hoc Networks," Proc. of the Second Annual Conference on Wireless on-Demand Network Systems and Services (WONS'05) Volume 00, 2005, pp 70-75.

[26] M.K. Denko., Chen Wei, "An architecture for integrating mobile ad hoc networks with the Internet using multiple mobile gateways, " Proc. of the Canadian Conference on Electrical and Computer Engineering, 2005, pp 1097-1102.

[27] Rakesh Kumar, Manoj Misra, Anil K. Sarje, "An Efficient Gateway Discovery in ad hoc Networks for Internet Connectivity, " Proc. of the International Conference on Computational Intelligence and Multimedia Applications 2007, pp 275-281.

[28] S.Y. Wang, "National Chiao Tung University Network Simulator," Network and System Lab, CSIE, NCTU, http://ns110.csie.nctu.edu.tw/, April 2003. 